\title{
Visual Abstraction for Games on Large Public Displays
}

\author{
David Gullick, Daniel Burnett, and Paul Coulton \\ Imagination Lancaster, LICA, Lancaster University, \\ LA1 4YW, UK. \\ \{d.gullick, d.burnett, p.coulton\}@lancaster.ac.uk
}

\begin{abstract}
From its earliest developments video game design has arguably been closely coupled to technological evolution particularly in relation to graphics. In very early games the limitations of technology led to highly abstracted graphics but as technology improved, abstraction has largely been left behind as developers strive towards ever-greater realism. Thus, games are generally drawing from conventions established in the mediums of film and television, and potentially limiting themselves from the possibilities abstraction may offer. In this research, we consider whether highly abstracted graphics are perceived as detrimental to gameplay and learnability by current gamers through the creation of a game using very low-resolution display that would accommodate a range of display options in a playable city. The results of trialing the game at a citywide light festival event where it was played by over 150 people indicated that abstraction made little difference to their sense of engagement with the game, however it did foster communication between players and suggests abstraction is a viable game design option for playable city displays.
\end{abstract}

Keywords: Game Design; Graphics; Abstraction; Realism, Resolution.

\section{Introduction}

While many game players will cite fun or gameplay as the most important attributes for a game, examining the marketing material produced by developers and publishers over many years might lead one to the conclusion it is primarily about graphics. This is because in its early days, games development were closely coupled to technological developments which were often intrinsically linked to notions such as Moore's Law [1]. The fidelity of the graphics was the simplest and quickest way that consumers could compare systems, and the complexity of graphic detail became the main area in which games would compete for customers. This concentration on graphics created a distinct change of style of the visual imagery, from abstraction to representation (often referred to as realism) [2,3].

Early video games relied heavily on abstraction in that they simplified visual assets to a few essentials and basic forms due to the very limited graphical capabilities of early arcade machines and consoles. These early games could be considered abstract in both appearance and behavior, because at the time of their development game interaction conventions were in their infancy.

With the development of the so-called third generation consoles we observed a shift to a concentration on representation, which seeks to create a resemblance and 
reproduce something; abstraction became more of an artistic choice than a technical default. This means there has been "a shift from perceptual abstraction to conceptual abstraction" [4].

Mark Wolf explains this shift in relation to art theorist Wilhelm Worringer, 1908 treatise, Abstraction and Empathy, within which he suggests that "there are two fundamental aesthetic impulses that are mutually exclusive, the desire for abstraction and the desire for empathy" [4]. This straightforward argument is that, as with Art, people will more readily engage with realism than abstraction, and this, in some way, explains the popularity of representational graphics within games.

Further it has been shown that a certain level of learnability can be beneficial in games [5], but how that level of learnability is achieved is different for every game. Modern games often rely on in-game tutorials to help increase the rate at which users can become familiar with a game, even though it is not always effective [6]. However, many older abstracted games such as Tetris allow users to learn through discovery whilst playing the game, which it has been argued gives the user a deeper understanding of the game as a whole [7].

In this research we consider whether rather than trying to avoid or sublimate abstraction, game design can usefully incorporate abstraction, resulting in new gaming experiences and game conventions which we believe would be relevant for creating a variety of flexible displays for a playable city of various resolutions.

\section{Game Design}

The game presented in this research is a two-player side scrolling game, similar in the style of early Atari favorites Defender and Chopper Command [8]. The player pilots the 'ship', only on the vertical axis, through a randomly generated tunnel avoiding obstacles and collecting power ups. The tunnel gets narrower and the number and complexity of obstacles increases the further down tunnel the player's progresses. Damage reduces the length of the ship whereas power ups increase its length, and the game is over when either of the players' ship's length reaches zero as shown in Figure 1.
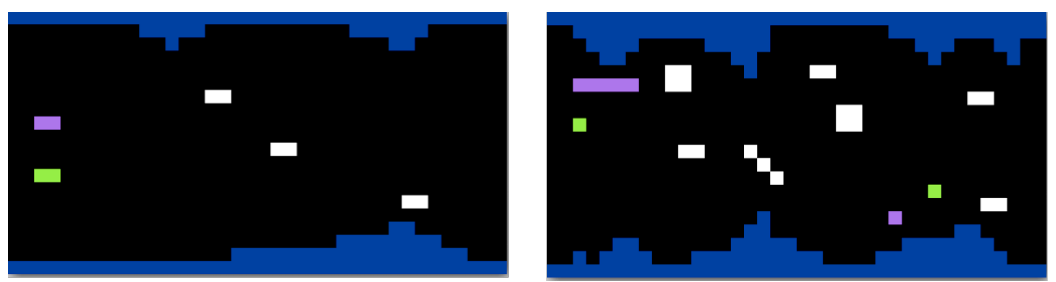

Figure 2. (a) Game Graphics. Left are Player 1 (purple), Player 2 (green), the tunnel walls (blue, top and bottom) and 3 'bullet' type enemies. (b) In this graphical representation of the screen, we can see player 1 has more life than player 2, and we can also see the 'block', 'spinning' and 'bullet' type enemies, and 2 power ups (right).

The game display exists as a large LED matrix of individually addressable RGB LEDs. Using a Teensy 3.1 each of the LEDs is addressable much like a pixel on a low 
resolution screen. From here, a small application running on a nearby computer duplicates a small area of a computer screen and forwards this to the teensy/LEDs. The screen itself has an extremely low resolution of 38 pixels wide by 20 high, and measures $122 \mathrm{~cm}$ wide by $70 \mathrm{~cm}$ high. The LEDS are covered by an acrylic diffuser, and the whole matrix is mounted on a stand that can be set as portrait or landscape (although we mainly used landscape). It is worth noting that this is a much lower resolution than would have been experienced in many of the early games. Additionally, the system is hooked up to a large speaker which plays background music in addition to audio for particular in game events (e.g. loose life, gain power up, hit wall, game over). In order for players to interact with the system, we provide two Leap Motions controllers (differentiated by the colored disks, shown in Figure 2) that limit user control to the vertical axis. When a user places their hand over the sensor, the players game character mimics the movement of the user's hand. Additionally, the players also have a large press button that they can use to start the game as shown in Figure 2 (b). As the players progress through the game, the frequency of enemies, their speed and the height and frequency of spikes on the walls increase, which makes the game more difficult. There is no 'win condition' for this game, but rather the game mechanic is to not be the first player to loose, or in other words, to 'beat' your opponent.
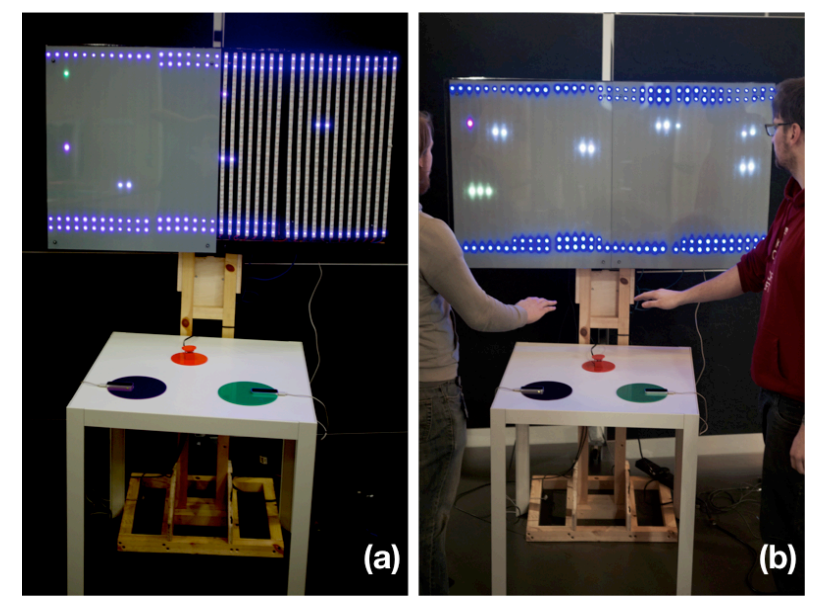

Figure 1: Abstracted Game

\section{User Trials}

Whilst we carried out small-scale play testing throughout the design process, the preliminary user trial was conducted at a local winter event called 'Light Up Lancaster'. Light Up Lancaster is a festival of lights held yearly in Lancaster City Center with numerous attractions and this game was one of these. In the space of 3.5 hours, the game was played by well over 150 people, with a wide range of ages from small children to pensioners. When no one was playing the game, the screen invited 
people to 'Press Start', which initially attracted people to the stand. Subsequently the crowds waiting to play seemed the main encouragement for new players to join in.

Working with such a low resolution creates a difficulty in implementing a tutorial for game learnability, thus we offered players a number of 'practice plays' before playing the game. Furthermore, designing the game in this way promoted discovery and exploration by players. Whilst players often needed an explanation in how to control the ships with the Leap Motion controller, they were able to quickly understand the basic principles. Interestingly though, many younger players seemed to want to touch the controller and some players would start moving their hand back and forward rather than up and down after playing the game for a while. Similarly, some players had initial difficulties with the automatic range calibration of the Leap Motion controller. If they began the game with their hand too close to the sensor, they would be unable to move their hand closer to the sensor in order to move the ship down. As the playing time of the games was short (typically 2-3 minutes max) we did not have any incidents of fatigue in terms of holding hand in an elevated position which is a design concern that should be considered for gesture control in games [9].

What was noticeably different about this more abstract game was the way in which players communicated with each other in order to fully understand all of the game mechanics. For example, it is not immediately obvious that the length of the player's ship represents their remaining lives, as this is not labeled. This resulted in the players collaborating in exploring the game mechanics. More so, those playing the game often would explain the hidden mechanics to those watching and waiting to play in the crowd of spectators. With a more traditional less abstracted or high-resolution games, all of these aspects would probably have been explained by in game text or more expressive graphics. Thus, as perhaps might be expected, abstract games are harder to learn initially and require more trial and error discovery by players. It is hard to determine if this difficulty is due to the abstraction within the game, or the reliance on 'learnability though discovery' as these two elements are tightly coupled.

\section{Conclusions}

Game development has always been intrinsically linked to continuing technological enhancements with graphics being the main beneficiary since they are an easy way to make a distinction between products in the eyes of the consumer. This means games design has largely missed the opportunity to consider abstraction conceptually as well as perceptually. Therefore, this research considers how using abstraction as a deliberate design choice, rather than a forced design constraint, affects the experience of players.

The results from the preliminary event are very encouraging considering the high numbers of players that played the game. Despite the difficulty in observation and recording, the numerous positive comments and returning players, showed that this form of abstracted game is both engaging and welcomed across a wide demographic. In fact, the abstraction was often considered part of the game challenge. Whether this success is solely due to the abstracted nature of the designed game is hard to determine, however it does suggest that the role of abstraction in video games has been underexplored, and encourages further work in the area. 


\section{Acknowledgements}

We thank all the early play testers at Lancaster Institute for the Contemporary Arts and players at Light Up Lancaster for their enthusiastic playing of the game. This work was supported by the RCUK Digital Economy project, Creating and Exploring Digital Empathy (Grant Reference EP/L003635/1).

\section{References}

1. Ernkvist, M. Down many times, but still playing the game: Creative destruction and industry crashes in the early video game industry 1971-1986. History of Insolvency and Bankruptcy 161, (2008).

2. Collins, S. Game graphics during the 8-bit computer era. ACM SIGGRAPH Computer Graphics, 32(2), (1998), pp.47-51.

3. Masuch, M. and Röber, N. Game graphics beyond realism: Then, now and tomorrow. In Level UP: Digital Games Research Conference. DIGRA, Faculty of Arts, University of Utrecht, (2004).

4. Wolf, M.J. Abstraction in the video game. The video game theory reader, 1, (2003), pp.4765.

5. Jørgensen, Anker Helms. "Marrying $\mathrm{HCI} /$ Usability and computer games: a preliminary look." Proceedings of the third Nordic conference on Human-computer interaction. ACM, (2004).

6. Andersen E, O'Rourke E, Liu YE, Snider R, Lowdermilk J, Truong D, Cooper S, Popovic Z. The impact of tutorials on games of varying complexity. InProceedings of the SIGCHI Conference on Human Factors in Computing Systems 2012 May 5 (pp. 59-68). ACM.

7. Ryan, William, and Martin A. Siegel. "Evaluating interactive entertainment using breakdown: Understanding embodied learning in video games."Proceedings of DiGRA. 2009.

8. Kent S. The Ultimate History of Video Games: from Pong to Pokemon and beyond... the story behind the craze that touched our lives and changed the world. Three Rivers Press, (2010).

9. Kim Y, Lee G, Jo D, Yang U, Kim G, and Park J. Analysis on virtual interaction-induced fatigue and difficulty in manipulation for interactive 3D gaming console. In Consumer Electronics (ICCE), (2011), pp 269-270. 